\title{
Mandatory COVID-19 vaccination for oral health professionals (OHPs) - Ethical appraisal
}

SADJ February 2021, Vol. 76 No. 1 p42 - p45

LM Mtolo', PD Motloba ${ }^{2}$, NH Wood ${ }^{3}$

\begin{abstract}
The recently detected South African variant, Covid-19, 501Y.V2 is more transmissible, though not virulent as the initial strain. ${ }^{1}$ The morbidity and mortality rates due to this variant have risen exponentially, putting huge pressure on the healthcare system, locally and globally.

As a consequence the South African government imposed hard lockdown measures (level 3) as a means to curb the pandemic. Governments around the world are scrambling to obtain and roll-out Covid-19 vaccination programs to save lives and livelihoods. Most developed countries have initiated inoculations, amid widespread misinformation and hesitancy.
\end{abstract}

Literature indicate that healthcare professionals (HCPs) are generally complacent and hesitant about vaccination. The COVID-19 uptake is likely to be suboptimal among HCPs and the general populations. The voluntary immunization program will commence in February 2021, and the Healthcare professionals will be among the first to be vaccinated.

It remains to be seen if this cohort will achieve the desired vaccinations rates. Failure for this influential group to vaccinate could derail the program and lead to failure to achieve herd immunity. In the face of emerging vaccine hesitancy among HCPs, should oral health professionals be compelled to vaccinate? Will any refusal by OHPs to be immunized be morally justified?

Oral health professionals in this context of this paper, represent all oral health professionals involved in the management dental patients.

Author affiliations:

1. Thembelihle M Mtolo: BTech (DT)(TUT), Senior Dental Technologist, Dental Laboratory, Sefako Makgatho Health Sciences University, South Africa.

2. Pagollang D Motloba: BDS (Medunsa), MPH (Epidemiology) (Tulane), Head, Department of Community Dentistry, School of Oral Health Sciences, Sefako Makgatho Health Sciences University. ORCID Number: 0000-0003-1379-7576

3. Neil H Wood: Department of Periodontology and Oral Medicine, School of Oral Health Sciences, Sefako Makgatho Health Sciences University.

ORCID Number: 0000-0001-8950-7999

Corresponding author: Pagollang D Motloba

Department of Community Dentistry, School of Oral Health Sciences,

Sefako Makgatho Health Sciences University.

Email: pagollang.motloba@smu.ac.za

Author contributions:

1. Thembelihle M Mtolo: Primary author - $30 \%$

2. Pagollang D Motloba: Second author $-50 \%$

3. Neil H Wood: Third author $-20 \%$
No singular ethical framework is held as sufficient to resolve these questions. This paper interrogates aspect of clinical ethics, including the Hippocratic Oath, principilism, public health ethics (Utilitarianism) and Kantian deontology, to tackle the questions raised. We conclude that OHPs have a moral duty to be vaccinated against Covid-19.

\section{INTRODUCTION}

South Africa's first batch of one million Covid-19 vaccines arrived in the country on Monday (1 February). Despite logistical challenges and the complexities of rolling out the vaccination efforts, it remains to be seen whether uptake will be commensurate to the effort and gravity of the pandemic. Frontline healthcare professionals will be among the first persons to receive the vaccine in South Africa. Unfortunately not all healthcare professionals want to be the first in line. Vaccine hesitancy could negatively impact the plan to build herd immunity by inoculating $67 \%$ (40 million) of the South African population. ${ }^{2}$

The acceptability of COVID-19 vaccines by healthcare professionals (HCPs) is critical to improve vaccination uptake by the public. ${ }^{3}$ The attitudes of health professionals about vaccines are an important determinant of the likelihood of patient's behavior regarding vaccination. This means that patients are more inclined to accept vaccination if their healthcare providers recommend it. ${ }^{4}$

Regrettably the literature indicates that healthcare professional's willingness to vaccinate is not significantly different from that of the general population. ${ }^{5}$ These statistics raise critical questions about the healthcare professional's intention to vaccinate, and the causes of their hesitancy. The low vaccination rates by healthcare workers, and by extension the population, represents the most serious global threat during this pandemic.

We ask if healthcare professionals should be obligated to vaccinate, more so that COVID-19 vaccination is currently not mandatory in South Africa. Can refusal of these frontline workers to be immunized be justified? More specifically, are OHPs, who are at increased risk of contracting COVID-19, obligated to vaccinate?

\section{Overview of current Covid-19 vaccines}

The extraordinary speed at which Covid-19 vaccines are developed is unpreceded and remarkable. ${ }^{6}$ Since April 
2020, over 115 vaccines were candidates of rapid development and clinical trials around the world. ${ }^{7}$ Very few of these vaccines have achieved clinically significant efficacy levels for mass inoculation.

Currently immunization is ongoing using Pfizer $®-B i o N T e c h$ COVID-19, Moderna ${ }^{\circledR}$ COVID-19 and AstraZeneca ${ }^{\circledR}$ Covid -19 vaccines. ${ }^{8}$ Pfizer $\AA$ and Moderna $\AA$ vaccines use mRNA technology and require subzero refrigeration. A two-dose regimen of Pfizer® BNT162b2 vaccine confers 95\% protection against Covid-19 in persons 16 years and older. ${ }^{9}$

The efficacy of the Moderna ${ }^{\circledR}$ vaccine (mRNA-1273) in preventing symptomatic COVID-19 disease is recorded to be $94.1 \% .^{10}$ The AstraZeneca $\AA$ vaccine uses adenovirus vectored technology, and is storable at higher temperatures $(2.2-7.8)^{\circ} \mathrm{C}$. This makes this vaccine easier and cheaper to distribute globally despite is lower efficacy $(70 \%)$.

Russian vaccines, the Sputnik V® (the first vaccine to be developed in the world) and EpiVacCorona ${ }^{\circledR}$ have attracted criticism from regulatory agencies leading to limited uptake globally. ${ }^{11}$ Despite the controversies surrounding the clinical trials, these vaccines showed $91.45 \%$ and $95 \%$ efficacy. ${ }^{12}$ These four vaccines seem to prevent the Covid-19 infections, however like all other vaccines, the risk of anaphylaxis remain a serious concern.

Currently many candidate vaccines are subject of clinical trials and could soon be rolled out, including: the Ad5nCOV13 and the Corona Vac ${ }^{14}$ (China); the NVX-CoV2373 vaccine $^{15}$ (by Novavax $®$, USA); the Ad26.COV2.S ${ }^{16}$ (Johnson and Johnson®, USA and Netherlands).

As the vaccine race continues, a "new" normal is imminent, but some countries are set to get there faster than others.

\section{Side-effects of COVID-19 vaccines}

Covid-19 virus has unstable genome, resulting in numerous and rapid mutations. ${ }^{14,17}$ This could mean that the efficacy of the vaccines may differ depending on the strain and country of production. ${ }^{18}$

Therefore the reported efficacies might not be conferred and sustained everywhere in the world. Conceivably, there might be a need for yearly vaccination to sustain the protection or booster immunity against the virus. ${ }^{19}$

Similarly, the side effects will be variable, depending on the vaccines used. The reported side-effects of Covid-19 vaccines are based on data from phase III clinical trials. Patients who participated in these trials presented commonly with pain from the injection site, fatigue, headache, muscle and join pains, chills and fever. ${ }^{20}$

These symptoms are not unique to Covid-19 vaccines and resolve rapidly. ${ }^{21}$ Few adverse events have been reported, such as lymphadenopathy, paralysis and anaphylaxis. ${ }^{22}$ The phase IV trial period provides an opportunity for the medical community to understand the long term effects and adverse events of the Covid-19 vaccines and how to manage them.

\section{Dental practice in the time of Covid-19}

Very few OHPs are reported to have contracted SARSCoV-2 in the dental practices. This is largely due to adherence to strict prevention protocols, including wearing of mask, sanitization, social distancing and ventilation. ${ }^{23}$ Additionally, most OHPs restricted their practices to management of emergency cases. However, oral health professionals work in close proximity with the patients, making the risk of contracting Covid-19, a real possibility - and possibly a matter of 'when', not 'if'. ${ }^{24}$ SARS-CoV-2 is present in saliva, droplets and aerosols which contaminate surfaces and objects in the dental practice and the virus can remain viable for up to 9 days.

The emergence of the new and highly transmissible variant presents a grave threat to the sustainability of many dental practices, unless herd immunity is achieved and the "new" normality is restored. ${ }^{25}$

Is there an ethical duty for OHPs to vaccinate, or not?

\section{a). Hippocratic Oath and Georgetown Mantra (Principilism)}

When a dentist accepts, without coercion, the responsibility to care for any patient, their individual rights assume a subservient position to the dentist-patient relationship. Based on their duty of care towards patients, they are obligated to act professionally and responsibly and protect the best interests of their patients. According to the Hippocratic Oath and Georgetown Mantra (Principilism): i) the primary obligation of OHPs is not to harm their patient "primum non nocere", or non-maleficence; (ii) promotion of patient's wellbeing or beneficence; (iii) respect patient's expectations and preferences or autonomy; and (iv) not to prejudice patients in any way (justice).

The argument for mandatory vaccination of OHPs is grounded on the following premises:

1. That an unvaccinated OHPs should not treat patients, as doing so poses huge risk to patient's health, including the risk of death. OHPs will be failing to protect their patients from harm; and will be engaging in unethical practice of non-maleficence. Patients are vulnerable and have to be protected from any form of harm including from their OHPs. OHPs should at all times, engage in activities that promote the wellbeing of their patients.

2. By participating in vaccination programs and ongoing phase IV surveillance trial, OHPs will be making an immense and informed contribution to common good. First, OHPs are best placed to understand their medical status and vaccines-related complications. This will enrich the quality in reporting and of data generated from the post-marketing trials.

Second, medical interventions have side effects, and OHPs' refusal to vaccinate themselves would be a failure to acknowledge this fundamental clinical reality and medical phenomenon. It would be hypocritical of OHPs not to be vaccinated, when the medicines or procedures they prescribe to unsuspecting patients 
may have unknown long term side-effects. OHPs are obligated to implement medical interventions that are supported by "some" evidence, including the Covid-19 vaccine.

Third, a paucity of data on the long-term effects of Covid-19 is likely to contribute to "omission bias". ${ }^{26}$ This is the tendency to favour inaction (no vaccination) than commission (inoculation) when either can cause harm. The benefit (utility, reciprocity, group beneficence) to harm ratio is greater for oral health professionals to persuade OHPs to vaccinate. It is hence unjustifiable for a OHPs to refuse to vaccinate, even when it feels safer.

Fourth, by choosing to immunize, OHPs will contribute to improved patients' attitudes and intention towards immunization. Patients are likely to emulate and take advice from their OHPs. This could lead to increased uptake and eventually realization of the program goal (herd immunity).

Fifth, the generalised refusal by OHPs to vaccinate could have a catastrophic effect on the management of the pandemic. Notwithstanding the widespread misinformation and falsehoods on the vaccines, the premises above offer cogent arguments for mandatory vaccination of OHPs. It would be prudent for dental associations to advocate for greater involvement of OHPs in the vaccination program. Dental practices should be readied as immunizations sites, and OHPs trained to immunize patients.

\section{b). Bentham's Utilitarianism}

The Utilitarian ethical frameworks replace the individual ends with public concerns. Contrary to the Hippocratic Oath and Principilism, Bentham asserts that the objective of any action is to achieve maximum utility. This means consequences of an action is the ultimate basis for any judgment about the rightness or wrongness of that conduct. Therefore the optimization of public prevention effort (e.g. intensification of vaccination program) is utilitarian for as long it prevents public transmission of the disease. Partif refers to this principle of utility maximization as the Group Beneficence Principle. ${ }^{27}$

Like communitarian approach, individuals have moral obligation to benefit the collective. It is hence expected that an individual's contribution and effort, will lead to positive outcomes for the collective. ${ }^{28}$ The same principle has been applied to healthcare facilities, by targeting all HCPs for the benefit of the collective. For example, the introduction of mandatory vaccination in dental practices as a condition of employment would result in Group Beneficence.

This effort should result in many HCPs immunised, leading to "greatest happiness or benefit for the greatest number". We argue that mandatory immunization policies support the professional duty to protect others including patients. This policy infringes the dentist's agency or autonomy, and violates the law. To implement such policies, one has to appeal to supererogation a plea to clinician to go beyond their call of duty.
Despite any associated ethical and moral contradictions, the implementation of mandatory vaccination has significantly reduced risks of diseases in healthcare institutions. ${ }^{29}$ It is therefore incumbent on policy makers to design programs that incentivise and encourage HCPs to vaccinate. ${ }^{30}$ According to Bentham's utilitarian morality, OHPs may not refuse to immunize. The consequences of such actions are far worse than the inoculation itself.

\section{c). Kantian Deontology}

Kantian deontology, establishes that acting according to "duty" and for "duty's sake" is a categorical imperative. ${ }^{31}$ This means an individual must act in accordance to maxim so that it becomes universal law (generalization test). ${ }^{32}$

To evaluate the morality of an act according to Kant's universal law, we ask - what if everybody acted in this way? What if OHPs everywhere refused vaccination, including for their close relations? Generalised refusal to vaccinate would certainly hinder the realization of herd immunity, which is not moral according to Kant.

Herd immunity might still be achieved if few OHPs don't vaccinate. However, the refusal of one dentist is one too many, and could lead to a "slippery slope" from the vaccination program might not recover. It is irrational for OHPs to refuse as a collective to contribute to building of herd immunity and protection other human being. It cannot be willed that such an action be universal law.

With regards to autonomy, Kant argues that in our pursuit for "greatest good" or positive outcomes, we should "never treat humanity as a means to an end, but as an end in itself". ${ }^{33}$ Whatever the ultimate positive consequences are pursued, individuals should not be harmed, i.e. virtuous ends should not justify unethical means. This means that the burden on individuals to vaccinate and contribute to herd immunity, should not come at an unreasonable and unfair cost. ${ }^{34}$

We ask, what cost could supersede the obligation to vaccinate? In other words what circumstances would make Covid-19 vaccination by OHPs, beyond the "call of duty" or supererogatory? Immunizations without proper medical support and consideration for cultural, religious and psychological factors could constitute high costs and conditions for exemptions. Beyond these reasons, the moral burden and grounds to reject vaccination are unjustifiable.

A dentist who receives medical support, and whose culture and religion are respected, may not have valid grounds not to vaccinate. Kant offers therefore, a strong persuasion why refusal of OHPs to vaccinate is not moral.

\section{CONCLUSION}

Voluntary vaccination programs or policies generally fail to achieve desired goals. Mandatory policies, although effective, may violate the principles of autonomy and individual rights. Amidst tensions between moral viewpoints, the evidence for mandatory vaccination remains strong.

This policy is clearly justified by the Hippocratic Oath, deontological approach, and the utilitarian standpoint. 
Ethical principles offer partial justification and the appeal to individual right to choose, as grounds for OHPs to refuse vaccination is inadequate. Therefore, OHPs as frontline workers, have been prioritized to receive doses of vaccines. Without any medical contraindications, these candidates have moral duty vaccinate and contribute to attainment of herd immunity. In so doing they will continue to provide essential services to their patients.

\section{References}

1. Erol A. Are the emerging SARS-COV-2 mutations friend or foe? Immunology Letters. 2020; 230: 63-4.

2. Randolph HE, Barreiro LB. Herd Immunity: Understanding COVID-19. Immunity. 2020; 52(5): 737-41.

3. Paterson P, Meurice F, Stanberry LR, et al. Vaccine hesitancy and healthcare providers. Vaccine. 2016; 34(52): 6700-6.

4. Asma S, Akan H, Uysal $\mathrm{Y}$, et al. Factors effecting influenza vaccination uptake among health care workers: a multi-center cross-sectional study. BMC infectious diseases. 2016; 16(1): $1-9$.

5. Kabamba Nzaji M, Kabamba Ngombe L, Ngoie Mwamba G, et al. Acceptability of Vaccination Against COVID-19 Among Healthcare Workers in the Democratic Republic of the Congo. Pragmat Obs Res. 2020; 11: 103-9.

6. Le TT, Andreadakis Z, Kumar A, et al. The COVID-19 vaccine development landscape. Nat Rev Drug Discov. 2020; 19(5): 305-6.

7. Corum J, Grady D, Wee S-L, et al. Coronavirus vaccine tracker. The New York Times 2020. Accessed at www.nytimes.com/interactive/2020/science/coronavirus-vaccinetracker.html on 8 January2021.

8. Lipsitch M, Dean NE. Understanding COVID-19 vaccine efficacy. Science. 2020; 370(6518): 763-5.

9. Polack FP, Thomas SJ, Kitchin N, et al. Safety and efficacy of the BNT162b2 mRNA Covid-19 vaccine. New England Journal of Medicine. 2020; 383(27): 2603-15.

10. Baden LR, El Sahly HM, Essink B, et al. Efficacy and safety of the mRNA-1273 SARS-CoV-2 vaccine. New England Journal of Medicine. 2020. https://doi.org/10.1056/NEJMoa 2035-389.

11. Callaway E. Russia announces positive COVID-vaccine results from controversial trial. Nature. 2020. https://doi.org/ 10.1038/d41586-020-03209-0.

12. De Soto JA. Evaluation of the Moderna, Pfizer/BioNtech, AstraZeneca/Oxford and Sputnik V Vaccines for COVID-19. 2020. https://doi.org/10.31219/osf.io/e4rqu.

13. Zhu F-C, Li Y-H, Guan X-H, et al. Safety, tolerability, and immunogenicity of a recombinant adenovirus type-5 vectored COVID-19 vaccine: a dose-escalation, open-label, non-randomised, first-in-human trial. The Lancet. 2020. https://doi. org/10.1016/S0140-6736(20)31208-3.

14. Palacios R, Patiño EG, de Oliveira Piorelli R, et al. DoubleBlind, Randomized, Placebo-Controlled Phase III Clinical Tria to Evaluate the Efficacy and Safety of treating Healthcare Professionals with the Adsorbed COVID-19 (Inactivated) Vaccine Manufactured by Sinovac-PROFISCOV: A structured summary of a study protocol for a randomised controlled trial. Trials. 2020; 21(1): 1-3.

15. Keech C, Albert G, Cho I, et al. Phase 1-2 trial of a SARSCoV-2 recombinant spike protein nanoparticle vaccine. New England Journal of Medicine. 2020; 383(24):2320-32.

16. Sadoff J, Le Gars M, Shukarev G, et al. Interim Results of a Phase 1-2a Trial of Ad26.COV2.S Covid-19 Vaccine. New England Journal of Medicine. 2021. https://doi.org/.

17. Knoll MD, Wonodi C. Oxford-AstraZeneca COVID-19 vaccine efficacy. The Lancet. https://doi.org/.

18. Tuite AR, Zhu L, Fisman DN, et al. Alternative dose allocation strategies to increase benefits from constrained COVID19 vaccine supply. Annals of internal medicine. 2021. https:// doi.org/.
19. Tegally $H$, Wilkinson E, Giovanetti M, et al. Emergence and rapid spread of a new severe acute respiratory syndromerelated coronavirus 2 (SARS-CoV-2) lineage with multiple spike mutations in South Africa. medRxiv. 2020. https://doi. org/10.1056/NEJMoa2034201.

20. Kostoff RN, Briggs MB, Porter AL, et al. [Comment] COVID19 vaccine safety. International journal of molecular medicine. 2020; 46(5):1599-1602.

21. Ledford $H$. Oxford Covid-vaccine paper highlights lingering unknowns. Nature. 2020; 588: 378-9.

22. Ather A, Patel B, Ruparel NB, et al. Coronavirus disease 19 (COVID-19): implications for clinical dental care. Journal of endodontics. 2020; 46(5): 584-95.

23. Passarelli PC, Rella E, Manicone PF, et al. The impact of the COVID-19 infection in dentistry. Experimental Biology and Medicine. 2020; 245(11): 940-4.

24. Meng L, Hua F, Bian Z. Coronavirus disease 2019 (COVID19): emerging and future challenges for dental and oral medicine. Journal of dental research. 2020; 99(5): 481-7.

25. Passarelli PC, Rella E, Manicone PF, Garcia-Godoy F, D'Addona A. The impact of the COVID-19 infection in dentistry. Experimental Biology and Medicine. 2020; 245(11): 940-4.

26. Jiménez ÁV, Mesoudi A, Tehrani JJ. No evidence that omission and confirmation biases affect the perception and recall of vaccine-related information. PloS one. 2020; 15(3):e022 8898. https://doi.org/10.1371/journal.pone.0228898.

27. Parfit D. Reasons and persons: Oxford University Publishers 1984.

28. Otsuka M. The paradox of group beneficence. Philosophy \& Public Affairs. 1991: 132-49.

29. Betsch C, Böhm R. Detrimental effects of introducing partial compulsory vaccination: experimental evidence. The European Journal of Public Health. 2016; 26(3): 378-81.

30. Dubov A, Phung C. Nudges or mandates? The ethics of mandatory flu vaccination. Vaccine. 2015; 33(22): 2530-5.

31. 30. Wood AW. Kant, Immanuel. International Encyclopedia of Ethics. 2013.

32. Giubilini A, Douglas T, Savulescu J. The moral obligation to be vaccinated: utilitarianism, contractualism, and collective easy rescue. Med Health Care Philos. 2018; 21(4): 547-60.

33. Wood A. Humanity as end in itself. Proceedings of the Eighth International Kant Congress 1995: 301-19.

34. Flanigan J. Mark Navin, Values and Vaccine Refusal: Hard Questions in Ethics, Epistemology, and Health Care. The Journal of Value Inquiry. 2017; 51(1):199-202. 